\title{
Remarks on Scalar Curvature and Concircular Field Equation
}

\author{
Ramesh Sharma* and Sharief Deshmukh \\ (Dedicated to the memory of Prof. Dr. Aurel BEJANCU (1946 - 2020))
}

\begin{abstract}
We show that the scalar curvature of a Riemannian manifold $M$ is constant if it satisfies (i) the concircular field equation and $M$ is compact, (ii) the special concircular field equation. Finally, we show that, if a complete connected Riemannian manifold admits a concircular non-isometric vector field leaving the scalar curvature invariant, and the conformal function is special concircular, then the scalar curvature is a constant.
\end{abstract}

Keywords: Scalar curvature, concircular vector field, concircular scalar equation, gradient Yamabe soliton.

AMS Subject Classification (2020): Primary: 53C21 ; Secondary: 53C24.

\section{Introduction}

One of the important problems in differential geometry is to seek distinguished Riemannian metrics, e.g. Einstein metrics (whose special cases are constant curvature metrics), and more generally, the constant scalar curvature metrics. Yamabe's theorem says that the metric of a compact Riemannian manifold can be conformally deformed to a metric of constant scalar curvature. In this note, we consider the scalar curvature as the solution of a concircular scalar equation, and show that the scalar curvature becomes constant in two cases.

In [6], Fialkow introduced the concept of a concircular vector field $V$ defined by the differential equation:

$$
\nabla V=\psi I
$$

on an $n$-dimensional connected Riemannian manifold $(M, g)$, where $\nabla$ denotes the Levi-Civita connection of $g$ and $\psi$ is a smooth function on $M$. This was used by B.Y. Chen [2] to provide a simple characterization of generalized Robertson-Walker spacetimes. In particular, if $V=D f$ for a smooth function $f$, where $D$ denotes the gradient operator of $g$ and $\nabla f$ denotes the 1-form $g$-equivalent to $D f$, then equation (1.1) assumes the form

$$
\nabla \nabla f=\psi g
$$

is called a concircular field equation. A non-constant solution of it is said to be a concircular field (Tashiro [10]). In particular, when $\psi=a f+b$ for constants $a$ and $b$, the equation (1.2) takes the form

$$
\nabla \nabla f=(a f+b) g
$$

and is called a special concircular field equation. It is to be noted that a complete Riemannian manifold satisfying (1.3) is isometric to the Euclidean space if $a=0$ [10], and the round sphere if $a$ is negative (Obata [8]) The equation (1.2) is equivalent to:

$$
L_{D f} g=2 \psi g
$$


where $L$ denotes the Lie-derivative operator. That is, $D f$ is a gradient conformal vector field with conformal scale function $\psi$. For details on this topic, we refer to Deshmukh and Al-Solamy [5].

From now on, $X, Y$, and $Z$ will denote arbitrary vector fields on $M$. The curvature tensor, Ricci tensor, Ricci operator and scalar curvature of $g$ will be denoted by $R, R i c$, $Q$ and $r$ respectively. Equation (1.2) can also be expressed as

$$
\nabla_{X} D f=\psi X .
$$

A natural question is to examine the situation when $f$ happens to be the scalar curvature $r$. Motivated by this question, we establish the following result.

Theorem 1.1. The scalar curvature of a Riemannian manifold $M$ is constant if (i) it satisfies the concircular scalar equation and $M$ is compact, (ii) it satisfies the special concircular scalar equation on any $M$ (not necessarily compact).

Now we recall (Daskalopoulos and Sesum [4]) that a gradient Yamabe soliton is a Riemannian manifold $(M, g)$ satisfying the condition

$$
\nabla \nabla f=(\lambda-r) g
$$

where $f$ is a smooth function (called the potential function) and $\lambda$ is a constant. The gradient Yamabe soliton is said to be shrinking, steady, or expanding when $\lambda>0,=0,<0$ respectively, and plays an important role in the study of the Yamabe flow: $\frac{\partial}{\partial t} g(t)=-r(t) g(t)$. The gradient Yamabe soliton is said to be trivial when $f$ is constant, in which case $r$ is constant. A compact gradient Yamabe soliton has constant scalar curvature, and hence trivial [4]. Chen and Deshmukh [3] obtained some conditions on Yamabe solitons to be of constant scalar curvature. As a consequence of part (ii) of Theorem 1.1 and definition (1.3), we obtain the following corollary.

Corollary 1.1. A gradient Yamabe soliton whose potential function is the scalar curvature $r$, is trivial, i.e. $r$ is constant.

Finally, we prove the following result.

Theorem 1.2. Let $(M, g)$ be an n-dimensional complete connected Riemannian manifold and $\xi$ be a concircular vector field on $M$ with nonzero conformal scale function $\psi$. If the scalar curvature $r$ is constant along the integral curves of $\xi$ and $\psi$ satisfies special concircular field equation, then ths scalar curvature is a constant.

\section{Proofs of Theorem 1.1}

For the part (i), we proceed by the hypothesis:

$$
\nabla_{X} D r=\psi X
$$

Its $g$-trace provides

$$
\psi=\frac{\Delta r}{n}
$$

Equation (2.1) shows that $D r$ is closed and conformal, i.e. $L_{D r} g=2 \psi g$. Hence, by a result ( Ros and Urbano [9] and Castro, Montealegre and Urbano [1]) we find that $Q(D r)=(1-n) D \psi$. Computing its divergence, and using (2.1) along with the twice contracted Bianchi identity: $\operatorname{div} \circ Q=\frac{1}{2} \nabla r$, leads to

$$
n|D r|^{2}+2 r \Delta r=2(1-n) \Delta \Delta r,
$$

where $\Delta$ is the Laplacian of $g$. Integration by parts shows that

$$
\Delta r^{2}=2\left(|D r|^{2}+r \Delta r\right) .
$$

Eliminating $r \Delta r$ between this equation and (2.3) provides

$$
(n-2)|D r|^{2}=-\Delta\left[r^{2}+2(n-1) \Delta r\right]
$$

As $M$ is compact, integrating the above equation and using Divergence theorem, immediately shows that $r$ is constant, proving part (i). 
Now For the part (ii), we have that $r$ is special concircular, i.e.

$$
\nabla_{X} D r=(a r+b) X .
$$

Computing $R(X, Y) D r$ through the above equation, and contracting it, gives $Q D r=a(1-n) D r$. Computing its divergence using (2.4) and twice contracted Bianchi second identity, we get

$$
|D r|^{2}=2(a r+b)[a n(1-n)-r]
$$

On the other hand, we see from (2.4) that $\nabla_{X}|D r|^{2}=2(a r+b) X r$. This, in conjunction with (2.5), yields

$$
\left[3 a r+2 b-n a^{2}+a^{2} n^{2}\right] D r=0 .
$$

So, either (i) $r$ is constant on $M$, or (ii) $D r \neq 0$ on an open dense subset $\mathcal{U}$ of $M$. In case (ii), equation (2.6) implies

$$
3 a r+2 b-n a^{2}+a^{2} n^{2}=0
$$

on $\mathcal{U}$. Thus, if $a=0$, then (2.7) implies $b=0$ and hence from equation (2.4), $D r=0$ on $\mathcal{U}$, a contradiction. Now, if $a \neq 0$, then (2.7) implies that $r$ is constant on $\mathcal{U}$, again a c ontradiction. Thus, $r$ is constant on $M$. This completes the proof.

\section{Proof of Theorem 1.2}

From the definition of concircular vector field it follows that

$$
R(X, Y) \xi=X(\psi) Y-Y(\psi) X,
$$

which gives $\operatorname{Ric}(X, \xi)=-(n-1) X(\psi)$, that is

$$
Q(\xi)=-(n-1) \nabla \psi
$$

where $\nabla \psi$ is the gradient of $\psi$.

Taking divergence on both sides of equation (3.1) we get

$$
-(n-1) \Delta \psi=\psi r+\frac{1}{2} \xi(r)=\psi r
$$

where we have used the fact that $r$ is a constant along the integral curves of $\xi$. Now, use that $\psi$ satisfies the concircular field equation, ( that is $\left.H_{\psi}=(a \psi+b) g\right)$, which gives

$$
\Delta \psi=n(a \psi+b) .
$$

Using above equation with equation (3.2), we get

$$
\left(a n+\frac{r}{n-1}\right) \psi+n b=0 .
$$

Differentiating it along $\xi$ and using that $r$ is constant along $\xi$ gives

$$
(\xi \psi)[r+n(n-1) a]=0
$$

At this point, we recall the following two properties of a concircular (i.e. closed conformal) vector fields (cf. [9, Lemma 1], see also [1]).

(1) The set $\mathcal{Z}(\xi)$ of zeros of $\xi$ is a discrete set.

(2) $|\xi|^{2} \nabla \psi=\xi(\psi) \xi$.

As the number of critical points of $\psi$ in the special concircular equation is at most 2, using the properties (1) and (2), we conclude that the zeros of $\xi \psi$ are discrete. Thus the continuity of $r$ and use of equation (3.3) show that $r$ is constant and equal to $-a n(n-1)$ on $M$, completing the proof.

Remark 3.1. Theorem 1.2 provides a generalization over an Einstein manifold with a concircular field, because, for Einstein case, equation (3.1) is $\nabla \psi=\frac{r}{n(1-n)} \xi$ and whose covariant derivative shows that Hess $\psi=\frac{r}{n(1-n)} g$ and so, $\psi$ is special concircular. 


\section{References}

[1] Castro, I., Montealegre, C. R. and Urbano, F.: Closed conformal vector fields and Lagragian submanifolds. Pacific J. Math. Complex Space Forms. 199, 269-302 (2001).

[2] Chen, B.Y.: A simple characterization of generalized Robertason-Walker spacetimes. Gen. Rel. Grav. 46, 1833 (5 pp.) (2014).

[3] Chen, B.Y. and Deshmukh, S.: A note on Yamabe solitons. Balkan J. Geom. Applns. 23, 37-43 (2018).

[4] Daskalopoulos, P. and Sesum, N.: The classification of locally conformally flat Yamabe solitons. Adv. Math. 240, 346-369 (2013).

[5] Deshmukh, S. and Al-Solamy, F.: Conformal gradient vector fields on a compact Riemannian manifold. Colloq. Math. 112, 157-161 (2008).

[6] Fialkow, A.: Conformal geodesics. Trans. Amer. Math. Soc. 45, 443-473 (1989).

[7] Hamilton, R.S.: The Ricci flow on surfaces, Mathematics and general relativity. Contemp. Math. 71, 237-262 (1988).

[8] Obata, M.: Conformal transformations of Riemannian manifolds. J. Differential Geom. 4, 311-333 (1970).

[9] Ros, A. and Urbano, F.: Lagrangian submanifolds of $C^{n}$ with confornal Maslov form and the Whitney sphere. J. Math. Soc. Japan. 50, 203-226 (1998).

[10] Tashiro, Y.: Complete Riemannian manifolds and some vector fields. Trans. Amer. Math. Soc. 117, 251-275 (1965).

\section{Affiliations}

RAMESH SHARMA

ADDRESS: University of New Haven, Dept. of Mathematics, CT 06514, USA.

E-MAIL: rsharma@newhaven.edu

ORCID ID: 0000-0003-3700-8164

\section{SHARIEF DESHMUKH}

AdDrEss: King Saud University, Dept. of Mathematics, Riyadh-19451, Saudi Arabia.

E-MAIL: shariefd@ksu.edu.sa

ORCID ID: 0000-0001-5026-8564 